\title{
The Use of Botulinum Toxin as a Resource for the Dental Practice
}

\section{Patricia Oyole-Maal*}

Department of Head and Neck Anatomy, MARC Institute, Miami, USA

*Corresponding author: Patricia Oyole-Maal, Department of Surgical Sciences, Department of Head and Neck Anatomy, MARC Institute, Miami, USA, Tel: +003057463534; E-mail: patricia.oyole@gmail.com

Received date: November 30, 2016; Accepted date: December 26, 2016; Published date: December 30, 2016

Copyright: (c) 2016 Oyole-Maal P. This is an open-access article distributed under the terms of the Creative Commons Attribution License, which permits unrestricted use, distribution, and reproduction in any medium, provided the original author and source are credited.

\section{Introduction}

Botulinum Toxin is a protein, which is the metabolite of the bacterium Clostridium botulinum. In our neural network, nerves that activate motor muscles and also some glands communicate with the muscles to trigger movement through acetylcholine, which is a neurotransmitter released into the muscles at the neuromuscular junctions.

In order to move, a muscle needs to receive a sign from the brain and following this sign the acetylcholine will be released into the muscle. This is a very basic concept necessary to understand how muscles work.

Botulinum Toxin is used as a medication because it has the ability to temporarily suspend the acetylcholine release from the motor nerves into the muscles, so when a muscle is treated with Botulinum Toxin it will present a partial limitation on its activity during a given period of time. The use of Botulinum Toxin as a drug is FDA approved [1] since 1989 to treat several conditions of the head and neck, including blepharospasm of the eyes, facial palsy, strabismus and cervical dystonia, among others (Figure 1).

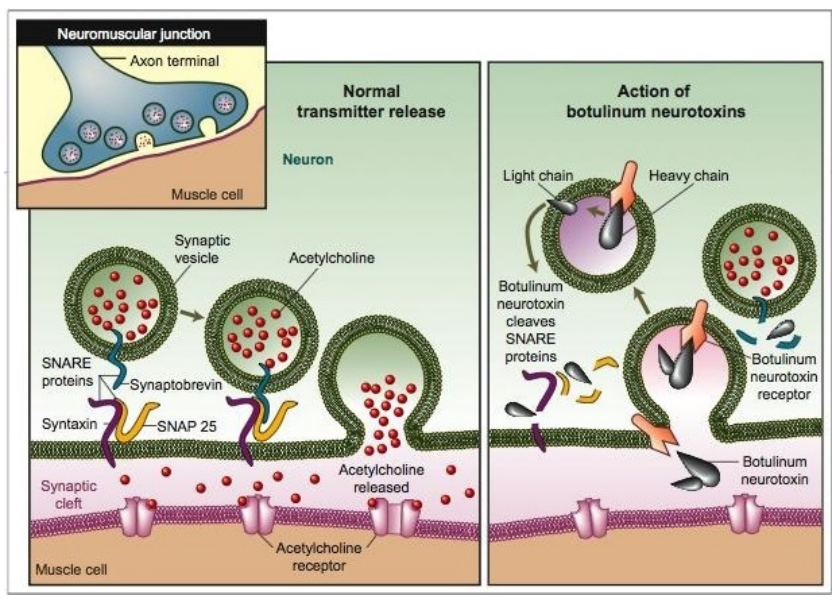

Figure 1: Neuromuscular junction.

Depending on the volume and concentration of Botulinum Toxin medication used, the limitation on the activity of the muscles treated will last from 3 to 6 months. When applied, Botulinum Toxin becomes part of a biochemical process that starts inside the muscle, and it will take 5 to 7 days to reach full effect, as the muscle receiving the treatment was soaked on its regular flow of acetylcholine, which will take a few days to wear off.
Mastication muscles, mimic and cervical muscles play a large role on the mechanics and function of the masticatory system. Other structures-like joints and ligaments-work alongside muscles to accommodate teeth and its functional activity.

On this thought, every time we identify a condition that is caused by inadequate muscle activity, there is an opportunity for Botulinum Toxin to be used as a resource to decrease muscle tone that may be harmful to the patient. Examples of that are hypertrophic muscles of the face and neck, hyperactivity of muscles of the mastication, of the chin and lips, and also psychosomatic triggered activity of mastication muscles, like clenching and bruxism [2].

At the dental practice, we have to treat a range of pathologies and many times dental conditions may be consequences of muscle activity. The FDA approval for the use of Botulinum toxin to treat mimic muscles was issued in 2002 for aesthetic purposes, and although this approval is not directly related to its use for masticatory muscles, since then it is been widely used as a resource to keep muscle activity from aggravating TMJ pathologies and disorders. The Dental Quality Assurance Commission (DQAC) of Washington has released an interpretive statement effective July 26 of 2013, which affirms the ability of general dentists to use Botulinum Toxin when "used to treat functional or aesthetic dental conditions and their direct aesthetic consequences" and also reinforcing that Continuing Education is available to provide training so "the treating dentist can have appropriate, verifiable training and experience" [3].

Dentists are the gatekeepers for a whole range of orofacial pains. Many times the exact source of the pain is hard to be identified given the number of structures that share sensory innervation and that are accumulated in very small spaces. Also, the overlapping of structures and reflex pain that are common factors for many structures in the head and neck, make the diagnosis of specific conditions very hard to reach.

Radiographic images, CT scans, anesthetics, percussion tests, electric pulp tests and other tools are daily used by Dentists to exclude or determine options in a wide range of pathologies, and for any differential diagnose study, all information is relevant, because based on the differential diagnose process, procedures will be selected to be performed and are expected to address the pain.

Dentists are expected to solve orofacial pain and deliver the best outcome available within the standards of care, and in the case of unsuccessful outcome, the liability risk is a relevant factor to be considered when we decide to move forward with procedures that are irreversible. Based on statistics of a systematic review published on JADA-December of 2011, "potentially more than 680,000 cases of tooth-related pain are diagnosed incorrectly each year". When after a throughout differential diagnosis process the pain is still not addressed, 
it is diagnosed as idiopathic orofacial pain, which while acceptable, it is a frustrating outcome both for the treating Doctor and for the patient.

Therefore, Botulinum Toxin is a useful resource when it comes to the differential diagnosis process, as it can decrease pain that may arise from inflammatory trigger points on mastication and cervical muscles, and point out either if the pain is muscle related or not. Since the medication will eventually wear off, no irreversible procedure is at stake. Also, the Botulinum Toxin as a timed action medication doesn't depend on patient cooperation, unlike mouth guards.

Because of the limitations we face daily with orofacial pain diagnosis; the actual consensus to treat idiopathic orofacial pain is the non-invasive, pharmacologic approach: the prescription of antiinflammatories and painkillers. Despite the reasonable results on pain management we can reach with this consensus, the palliative feature of painkillers and the fact that patients create resistance to some drugs have contributed to the actual epidemic of addiction that the American government and Health Department are enduring with hardship (Figure 2).

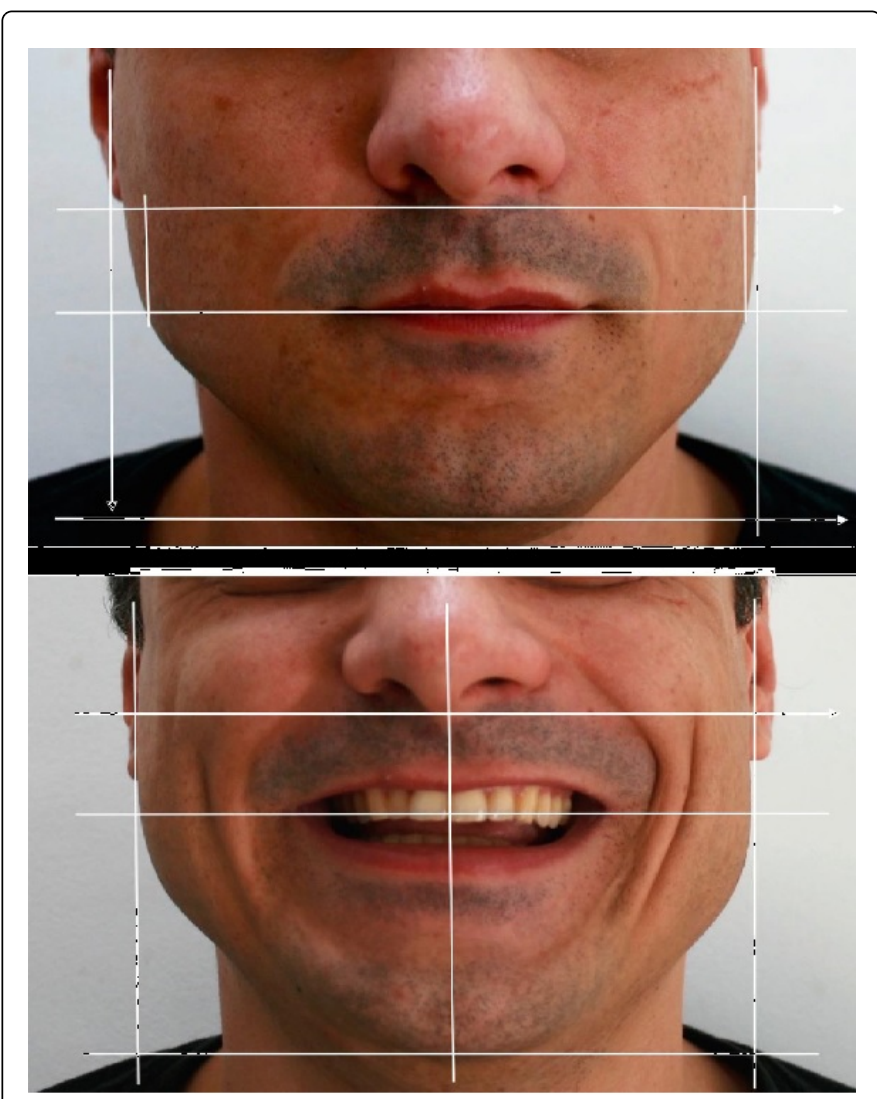

Figure 2: Patient with orofacial pain of unknown origin. Clinically, presents hypertrophic mastication muscles. Masseter on left side of the face presents enlarged and hypertonic while on rest. Received treatment with botulinum toxin on 3 trigger points found on left masseter muscle and felt significant relieve of the pain 6 days after the treatment.
Hence, adding Botulinum Toxin to the set of tools used in order to provide the best quality of healthcare to patients-either if only as a diagnostic tool or also as a treatment resource-is a decision that should be considered by Dentists willing to improve their quality of care.

\section{References}

1. Food and Drug Administration approvals.

2. Benjamin P (2011) Pain after routine endodontic therapy may not have originated from the treated tooth. J Am Dent Assoc 142: 1383-1384.

3. Dental Quality Assurance Commission Interpretive statement. 